\title{
Development of web-based dynamic nomogram to predict survival in patients with gastric cancer: a population-based study
}

\author{
Atefeh Talebi \\ Iran University of Medical Sciences \\ Nasrin Borumandnia ( $\nabla$ borumand.n@gmail.com ) \\ Shahid Beheshti University of Medical Sciences \\ Somayeh Abbasi \\ Islamic Azad University \\ Hassan Doosti \\ Macquarie University \\ Mohamad Amin Pourhoseingholi \\ Shahid Beheshti University of Medical Sciences \\ Shahram Agah \\ Iran University of Medical Sciences \\ Seidamir Pasha Tabaeian \\ Iran University of Medical Sciences
}

\section{Research Article}

Keywords: Gastric cancer, Dynamic nomogram, Cox proportional hazard regression model, Survival analysis

Posted Date: September 20th, 2021

DOI: https://doi.org/10.21203/rs.3.rs-902685/v1

License: (c) (1) This work is licensed under a Creative Commons Attribution 4.0 International License. Read Full License

Version of Record: A version of this preprint was published at Scientific Reports on March 17th, 2022. See the published version at https://doi.org/10.1038/s41598-022-08465-w. 
Development of web-based dynamic nomogram to predict survival in patients with gastric cancer: a population-based study

\author{
Atefeh Talebi ${ }^{1}$, Nasrin Borumandnia ${ }^{2 *}$, Somayeh Abbasi ${ }^{3}$, Hassan Doosti ${ }^{4}$, Mohamad Amin \\ Pourhoseingholi $^{5}$, Shahram Agah ${ }^{6}$, Seidamir Pasha Tabaeian ${ }^{1, * *}$ \\ 1. Biostatistician, Colorectal Research Center, Iran University of Medical Sciences, Tehran, Iran \\ 2. Urology and Nephrology Research Center, Shahid Beheshti University of Medical Sciences, \\ Tehran, Iran
}

3. Department of Mathematics, Isfahan (khorasgan) Branch, Islamic Azad University, Isfahan, Iran

4. Department of Mathematics and Statistics, Macquarie University, Sydney, Australia

5. Gastroenterology and Liver Diseases Research Center, Research Institute for Gastroenterology and Liver Diseases, Shahid Beheshti University of Medical Sciences, Tehran, Iran

6. Professor of Internal Medicine \& Gastroenterology, Colorectal Research Center, Iran University of Medical Sciences, Tehran, Iran

2,* Corresponding Author: Urology and Nephrology Research Center, Shahid Beheshti University of Medical Sciences, Tehran 1666663111, Iran, borumand.n@ gmail.com

Telephone: +98-21- 22770954

1,** Co-corresponding Author: Gastroenterology and Hepatology, Iran University of Medical Sciences, Tehran, Iran. aptabaiyan@gmail.com

Telephone: +98-21- 66554790

Fax: +98-21- 66554790 


\begin{abstract}
Gastric cancer (GC) is the fifth most frequent malignancy worldwide and the third leading cause of cancer-associated mortality. The study's goal was to construct a predictive model and nomogram to predict the survival of GC patients. This historical cohort study assessed 733 patients who underwent treatments for GC. The univariate and multivariable Cox proportional hazard $(\mathrm{CPH})$ survival analyses were applied to identify the factors related to overall survival (OS). A dynamic nomogram was developed as a graphical representation of the $\mathrm{CPH}$ regression model. The internal validation of the nomogram was evaluated by Harrell's concordance index (C-index) and time-dependent AUC. The results of the multivariable Cox model revealed that the age of patients, body mass index (BMI), grade of tumour, and depth of tumour elevate the mortality hazard of gastric cancer patients $(\mathrm{P}<0.05)$. The built nomogram had a discriminatory performance, with a C-index of 0.64 (CI: $0.61,0.67)$. We constructed and validated an original predictive nomogram for OS in patients with GC. Furthermore, nomograms may help predict the individual risk of OS in patients treated for GC.
\end{abstract}

Keywords: Gastric cancer, Dynamic nomogram, Cox proportional hazard regression model, Survival analysis. 


\section{Introduction}

Gastric cancer (GC) is one of the most common malignancies, with high incidence and mortality rates, and ranks as the fifth most frequent cancer and the third leading cause of cancer-related deaths globally ${ }^{1}$. The cancer is most commonly diagnosed in men among five countries (Bhutan, China, Kyrgyzstan, Mongolia, and Vietnam) ${ }^{2}$. Despite the fact that the incidence of GC has reduced during the past decades in European countries, the prognosis stays poor ${ }^{3}$. Also, the 5-year overall survival rate for GC patients is approximately 25 percent in the West ${ }^{4}$. Though the incidence of GC has decreased in Western countries in recent decades, its incidence has remained high in some Eastern countries (e.g., China, Japan, and Korea). Basis on the cancer registry of Turkey, GC may be the 5th most common malignancy in both men and women ${ }^{5}$. countries, twothirds of new cases of stomach cancer are recorded, and survival is still low in both developed and developing countries ${ }^{6}$.

The incidence is approximately 7300 cases per year among the Iranian population, which is the most common cancer in men ${ }^{7}$. Moreover, mortality from GC can be the first leading factor of death due to cancer in both sexes ${ }^{8}$. However, the incidence of the disease was higher in men than in women ${ }^{9}$. Considering the low rate of 5-year survival of GC patients, identification and control of predictive factors remain the essential prevention methods ${ }^{10}$.

Notwithstanding these technologies and therapeutic strategies have progressed over the last several decades, but GC patients' survival and risk factors remain unsatisfactory. It is crucial to recognize prognostic factors for those patients.

According to various statistical analyses, many researches have been performed to evaluate the prognosis factor on the survival of patients with GC ${ }^{10,11}$. Those methods incorporate several variables to predict a particular endpoint using traditional statistical methods (e.g., logistic or $\mathrm{CPH}$ regression models) ${ }^{12,13}$. Other studies have surveyed machine learning methods, which are a branch of artificial intelligence (AI) and computer science, on GC data ${ }^{14-17}$. Almazat et al. applied the Kaplan-Meier method to estimate overall survival in patients with GC ${ }^{18}$. The Cox and Frailty models of survival analysis were used and then compared with $\mathrm{C}$-index ${ }^{13}$. Other researchers have presented nomograms, which are the graphical representation to intuitive perception of clinicians in Colorectal cancer (CRC) and GC patients ${ }^{11,19,20}$. A nomogram, a simple graphical visualization combining and quantifying all independent prognostic factors, plays an increasingly vital role in medical sciences and clinical studies ${ }^{21}$. Moreover, nomograms play a significant role in improving prognostic accuracy by combining all independent prognostic factors and quantifying their risks $^{22,23}$.

The objective of this study was to establish a CPH regression model to explore prognosticators for OS in patients with GC. Then, a more dynamic nomogram was constructed to predict overall survival based on a relatively large historical cohort of patients with GC.

\section{Materials and methods}

\section{Data sources and ethics statement}


The demographical and clinicopathological data were extracted from a tertiary University-Hospital of Iran, Taleghani Hospital in Tehran, between 2013 and 2020. The data from Tehran city ( $n=733$ ) was regarded. The Ethics Committee of Iran University of Medical Sciences approved the study (Ethical code: IR.IUMS.REC.1399.122). We obtained the patients' informed consent to be allowed to use their medical information. The methods were carried out in accordance with the relevant guidelines and regulations.

\section{Demographic and clinical variables}

Survival time, based on months elapsed from the cancer diagnosis until death, was considered the outcome variable. The demographical and clinical variables, including sex, marital status, smoking status, body mass index (BMI), family history, type of treatment, grade of tumour, depth of tumour, number of involved lymph nodes were predictors. The patients' survival status was collected based on alive or dead.

\section{Statistical analysis}

The continuous variables were described as Mean \pm SD. Also, the frequency and percentage of categorical variables were reported. Missing data were imputed by fully conditional specification

${ }^{24}$. The Kaplan-Meier was used in order to estimate the survival function. We applied a univariable CPH model to explore the relationship between a patient's survival and explanatory variables. The selected variables with $\mathrm{P}<0.2$ in the univariable analysis were subjected to multivariable regression modelling. Then, the nomogram was illustrated according to the multivariable $\mathrm{CPH}$ model. At last, $\mathrm{C}$-index and time-dependent area under the roc curve were calculated to assess the internal validation. Also, internal calibration using bootstrap resampling was assessed by plotting the predicted probabilities from the model versus actual survival probabilities. The analysis was perform using the SPSS 23 and Stata 11. The survival, DynNom, rms, and hdnom packages in R 4.1.0 software were used to create a dynamic nomogram and to perform validation and calibration.

\section{Results}

The study population consisted of 733 confirmed patients with GC who underwent treatment. The median of follow-up time is 9.55 months ( $\mathrm{IQR}=4-19.13$, Range= 0.1-84). The Mean $\pm \mathrm{SD}$ age of patients was $59.49 \pm 13.47$ years, with ranges from 14 to 89 years. $932(69.1 \%)$ of patients were male, and 417 (30.9\%) were female. Six hundred ninety-nine (51.8\%) patients were censored, and $643(47.7 \%)$ of patients died at the end of follow-up. Other demographic and pathological characteristics of GC patients are given in Table 1. Also, the table revealed factors related to survival rate according to the univariable $\mathrm{CPH}$ regression model. 
Table 1. Univariable Cox regression analyses for survival in patients with GC

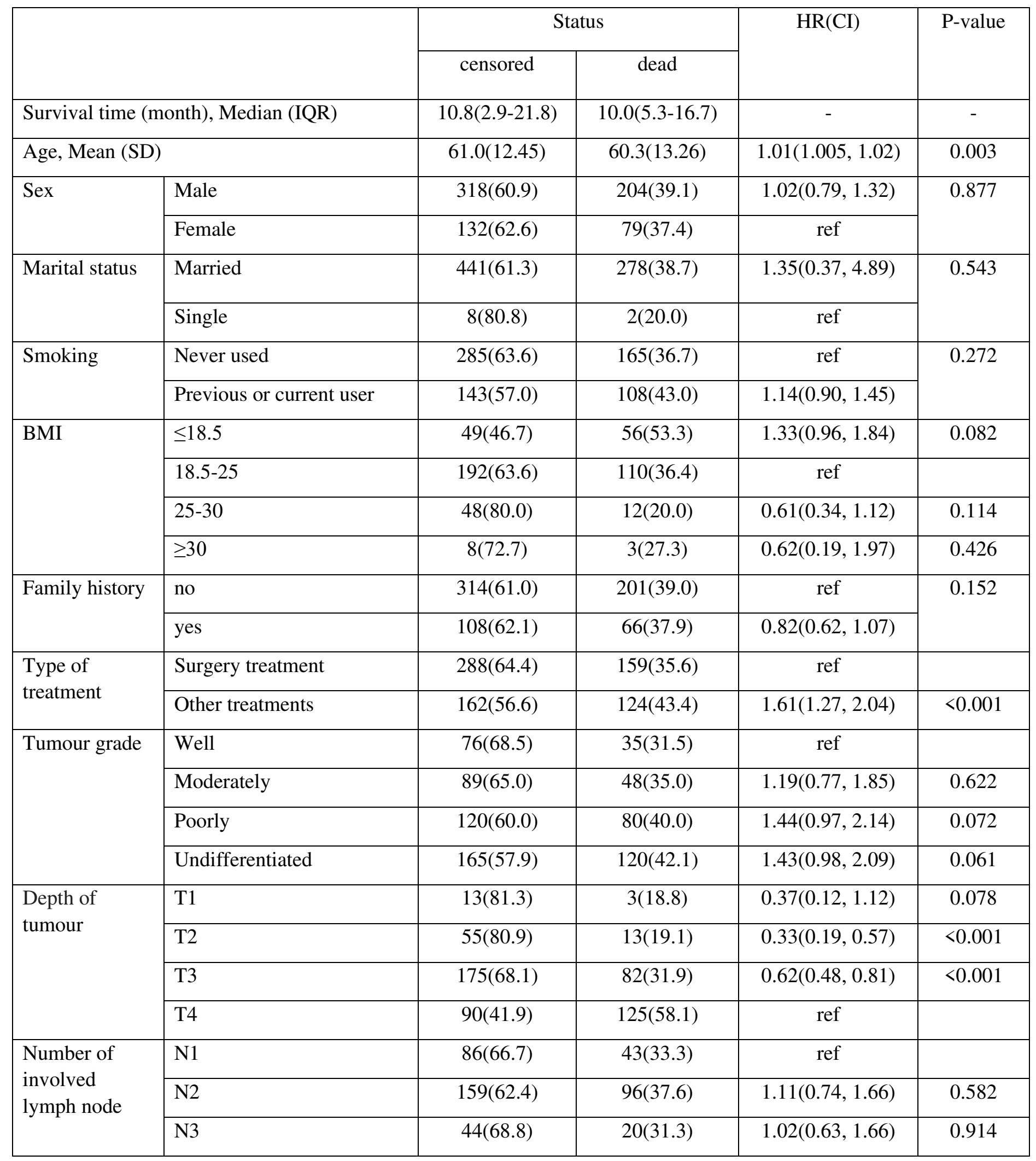


The results showed that age of diagnosing, BMI, family history, type of treatment, grade of tumour, and depth of tumour were significant in the univariable $\mathrm{CPH}$ model $(\mathrm{P}<0.05)$.

With regard to Kaplan-Meier plot, the 1-year survival rate was 70.9\%, while 3-year and 5-year survival rates were at $36.4 \%$ and $19.7 \%$, respectively.

The results of the multivariable $\mathrm{CPH}$ were presented in Table 2. Variables that had $\mathrm{P}<0.2$ in the univariable analysis were candidates for the multivariable regression analysis. The table showed that age of diagnosis, BMI, grade of the tumour, and depth of tumour are significant in the multivariable $\mathrm{CPH}$ model $(\mathrm{P}<0.05)$.

Table 2. Multivariable Cox regression model for survival in GC patients.

\begin{tabular}{|c|c|c|c|}
\hline & & $\mathrm{HR}(\mathrm{CI})$ & P-value \\
\hline Age & & $1.01(1.005,1.02)$ & 0.003 \\
\hline $\mathrm{BMI}$ & $\leq 18.5$ & $1.27(0.97,1.66)$ & 0.075 \\
\hline & $18.5-25$ & Ref & \\
\hline & $25-30$ & $0.54(0.32,0.90)$ & 0.020 \\
\hline & $\geq 30$ & $1.20(0.69,2.08)$ & 0.518 \\
\hline Family history & No & ref & \\
\hline & Yes & $0.93(0.70,1.23)$ & 0.629 \\
\hline Type of treatment & Surgery treatment & Ref & \\
\hline & Other treatments & $1.33(0.99,1.78)$ & 0.057 \\
\hline Tumour grade & Well & $1.04(0.69,1.56)$ & 0.853 \\
\hline & Moderately & $1.22(0.84,1.76)$ & 0.281 \\
\hline & Poorly & $1.57(1.14,2.15)$ & 0.005 \\
\hline & Undifferentiated & ref & \\
\hline Depth of tumour & $\mathrm{T} 1$ & $0.38(0.15,0.95)$ & 0.039 \\
\hline & $\mathrm{T} 2$ & $0.39(0.22,0.68)$ & 0.001 \\
\hline & $\mathrm{T} 3$ & $0.66(0.49,0.90)$ & 0.009 \\
\hline & $\mathrm{T} 4$ & Ref & \\
\hline
\end{tabular}


The result showed that for every ten years of increasing the age, the hazard rate increases by $10 \%$ (HR=1.01, P-value $<0.05)$. The HR in patients with the overweight range was $46 \%$ less the than normal group ( $\mathrm{HR}=54 \%, \mathrm{P}<0.05)$; however, obese patients had higher HR than normal weight, which is non-significant $(\mathrm{HR}=1.2, \mathrm{P}=0.518)$.

Also, the hazard ratio in patients who underwent chemotherapy, radiotherapy, and immunotherapy, presented as other treatments in the table, is 33\% more than the people who had surgery; however, the type of treatment was non-significant ( $\mathrm{HR}=1.33, \mathrm{P}=.057)$. Moreover, $\mathrm{HR}$ of tumour grade in patients with undifferentiated tumour grade was $57 \%$ more than people with well grade ( $\mathrm{HR}=1.57$, $\mathrm{P}<0.05$ ). When the depth of the tumour deteriorated, the HR was soared significantly in GC patients. Thus, the higher the tumour depth, the higher $\mathrm{HR}(\mathrm{P}<0.05)$.

The results of the multivariable CPH model were presented as a nomogram in Figure 1. The probability of survival for a GC patient can be predicted at a specific time point using this nomogram. The patient's characteristics have been plotted on each variable axis. To predict the survival probability of a patient, a vertical line is drawn from the patient's characteristics value to the top points scale. In this way, the number of points that were assigned by that variable value is determined. Then, the points from each variable value are summed. Finally, the sum on the total points is vertically projected onto the bottom axis, and a personalized probability survival time is obtained. Figure 2 shows the image of a web-based nomogram which is accessible in the https://nbshiny.shinyapps.io/GastricDynNom/. 


\section{Nomogram}

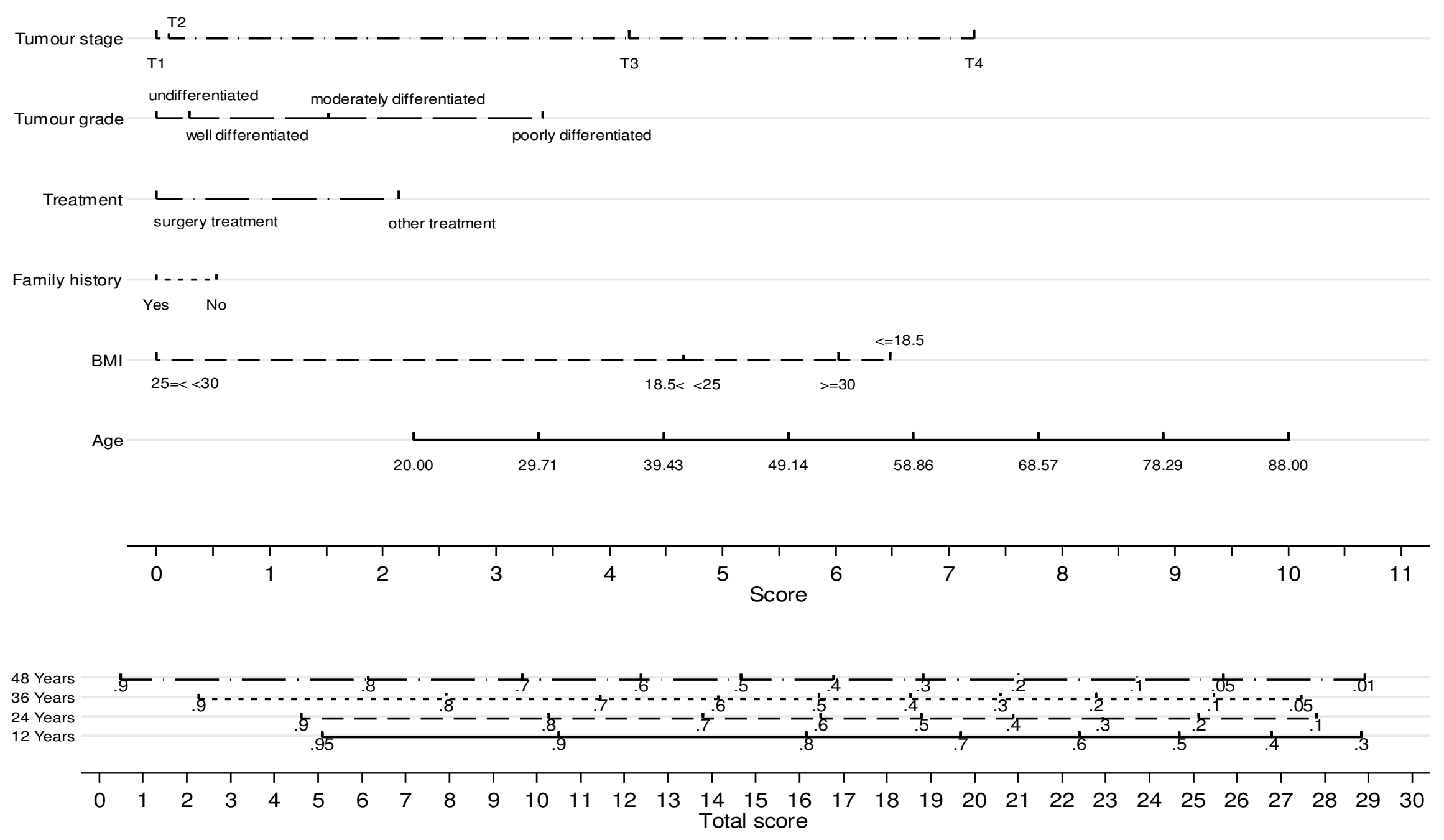

Fig 1. Predictive nomogram for survival in Gastric cancer patients. 

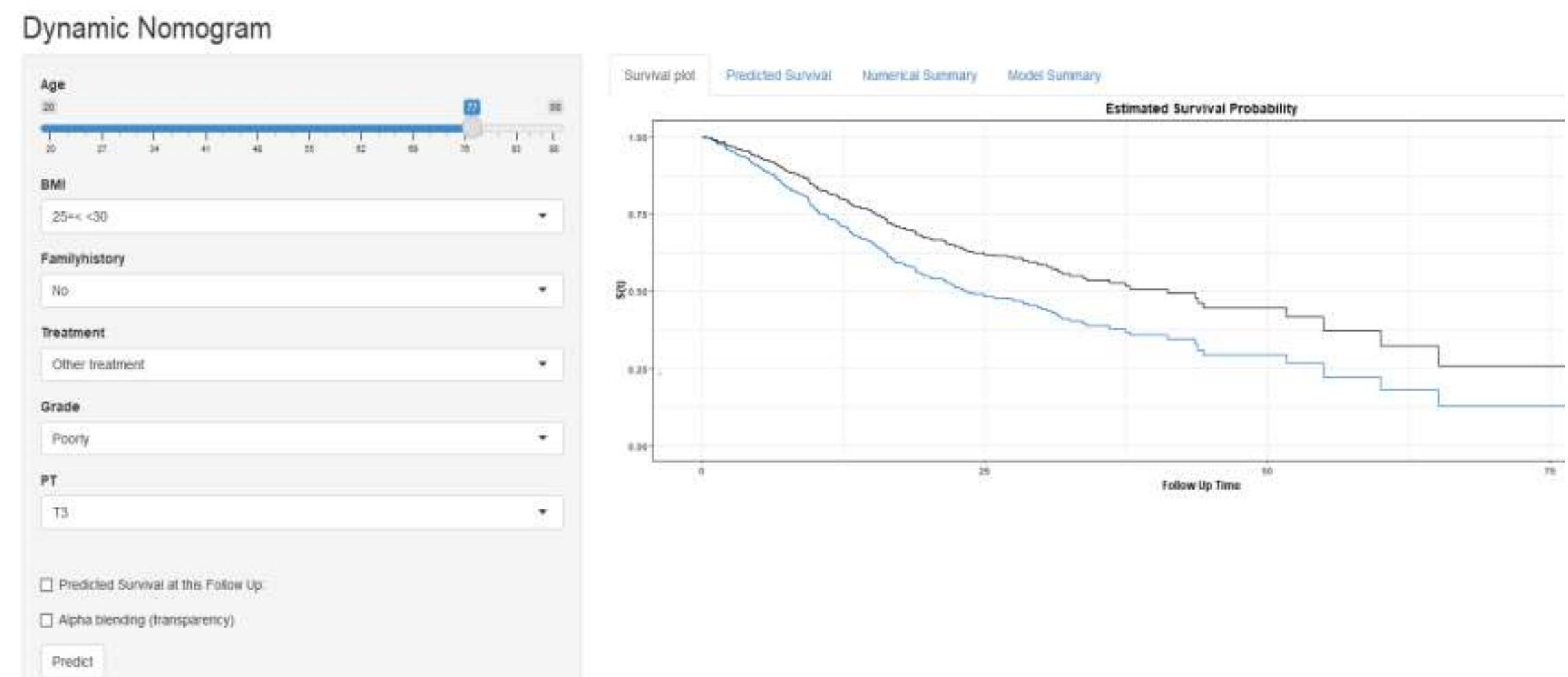

Fig 2. Web-based survival rate calculator (Dynamic Nomogram (shinyapps.io)) to predict the survival of GC patients; Two patients who are 77 years old, no surgery, no family history, PT3 and 25=<BMI<30, but have different grade, well (black line) and poorly (blue line), according to the web survival rate calculator. 


\section{Internal Validation and Calibration}

The internal validation was checked using C-Index and time-dependent AUC at evaluation time points. The C-index was calculated as 0.64 (CI: 0.61, 0.67). also, we validate the performance of the $\mathrm{CPH}$ model with bootstrap resampling every year from the first year to the sixth year. The time-dependent AUC at 1, 2-, 3-, 4-, 5- and 6-years follow-up have been presented in figure 3 (upper left). In addition, the internal calibration using bootstrap resampling was assessed by plotting the predicted probabilities from the model versus actual survival probabilities. In this way, the samples were split into ten risk groups, and the survival probabilities at 1 and 2 years were obtained and summarized as calibration plots in figure 3 (right).

\section{Time-dependent AUC}

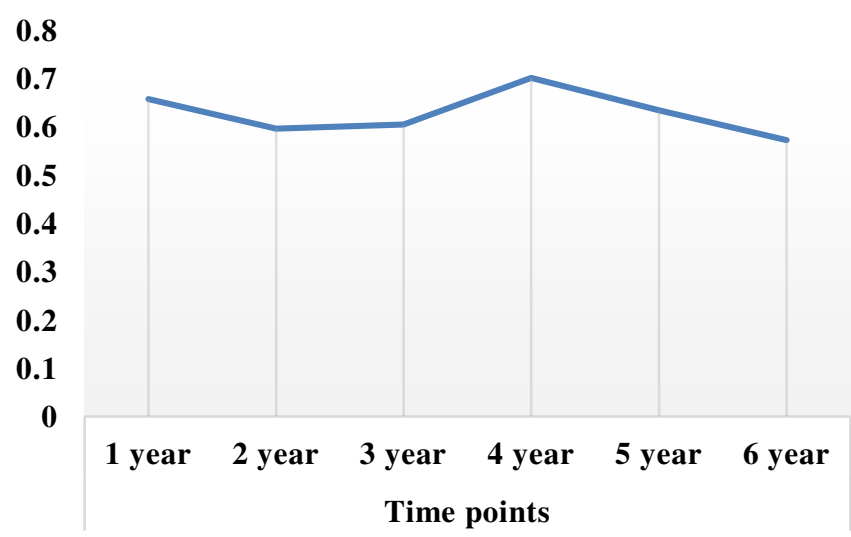

Fig 3. Time-Dependent AUC Summary at Evaluation Time Points (upper left). Internal calibration using bootstrap resampling at 1 year (lower left) and 2 years (lower right).
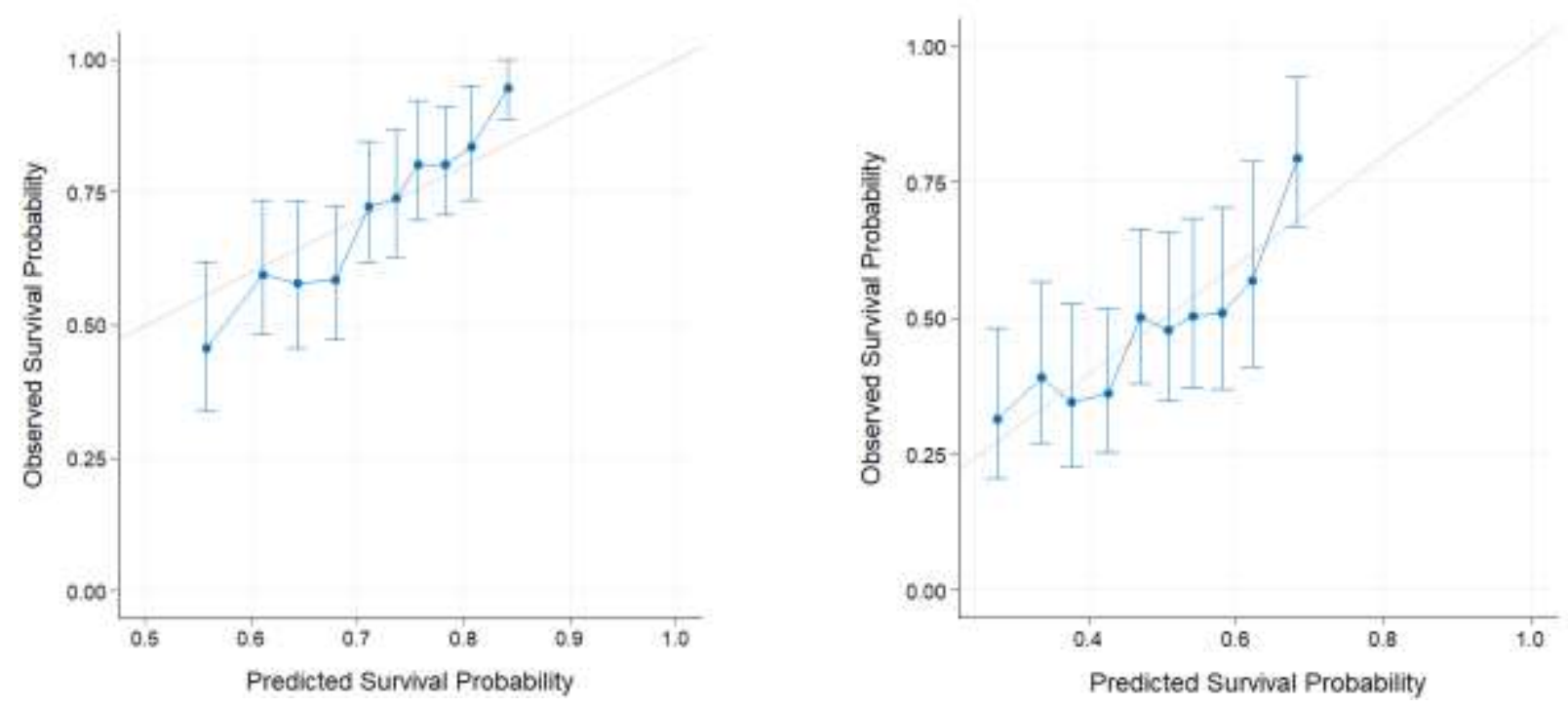

\section{Discussion}

This study provided a significant contribution through the use of a historical cohort of patients with GC who were treated in Iran from 2009 to 2020. The multivariable CPH model presented that 
age of diagnosing, BMI, family history, type of treatment, grade of tumour, and depth of tumour were statistically significant. Furthermore, we construct a nomogram to predict OS, which could provide individualized estimates of potential survival and aid individualized management decisions for GC. The C-index was applied to evaluate the model internal validation, and found that the prognostic model has high accuracy.

Nomogram is a precise and useful clinical tool that can help clinicians predict the probability of an outcome event, that is, survival time. A variety of nomograms have been built to predict the therapeutic benefits, the postoperative survival rate in patients with $\mathrm{GC}^{25-27}$. Here, we constructed a nomogram to predict the survival rate in GC patients. According to previous studies, a $\mathrm{C}$-index $>0.6$ indicated that the built model had an acceptance accuracy ${ }^{28-30}$. The value of this index was consistent with our study. In general, a few studies have applied AUC to predict the OS ${ }^{25,27,31}$. The AUC values of ROC were more than $60 \%$ for 1-, 3-, and 5-year of survival, which are compatible with our study.

Our multivariable $\mathrm{CPH}$ regression model discovered that age of diagnosis, BMI, grade of the tumour and tumour depth were independent risk factors in GC. Most of the previous studies focused on independent variables associated with GC and found that tumour depth, differentiation grade, size, and lymphatic invasion were closely associated with patients' survival ${ }^{32,33}$. Lu et al. reported age, differentiation, tumour size, number of examined lymph nodes, $\mathrm{pT}$ stage, $\mathrm{pN}$ stage, and adjuvant chemotherapy as associated with $\mathrm{GC}^{29}$. They showed that the hazard ratio increased with age, which means that, the older patients get, the higher the hazard. Similarly, our study found that age and pT stage were significant in the $\mathrm{CPH}$ model. In our multivariable $\mathrm{CPH}$ regression model, the groups with $25 \leq \mathrm{BMI}<30$ had significantly higher than the group with a BMI $\leq$ $18.5 \mathrm{~cm}$, suggesting that the BMI was a powerful predictor in patients with GC. The built nomogram finally corroborated that the BMI was one of the main risk factors in predicting survival of GC patients ${ }^{26,30}$. Regarding the grade of tumour, most previous studies reported that the tumour grade was a main predictor for patients with GC ${ }^{14,15,22,25,26,28,29}$. Similarly, our study found the grade of tumour can be a strong predictor in patients with GC. That means the worse the tumor grade, the greater the hazard ratio.

\section{Study limitation}

The key strength of this study is the long-term follow-up period. Also, we had several limitations. First, some variables, such as Helicobacter pylori infection status, location of tumour, demarcation line of tumour lesion, tumour markers, nutritional status, and Charlson Comorbidity Index, may also be potential risk factors in patients with GC and need to be incorporated into our model. Second, Statistical analysis was performed using internal validation. It is suggested that in future studies, external validation can be performed using another test dataset.

\section{Conclusion}

We successfully established a novel nomogram using patient data from the GC database in Taleghani University-Hospital. Furthermore, the age at diagnosing, BMI, tumour grade, depth of tumour made a significant contribution in predicting OS of patients with GC. 
Author Contributions: Conceptualization, A.T. and N.B.; methodology, A.T. and N.B.; software, N.B., A.T.; formal analysis, N.B. and A.T.; investigation, A.T.; resources, A.T.; responsible for data collection, M.A.P.; data curation, A.T.; N.B.; and S.A.; writing-original draft preparation, A.T., N.B.; writing-review and editing, A.T., N.B., S.A., H.D., S.A.P.T.; visualization, N.B. and A.T.; supervision, A.T., N.B., Sh.A., H.D.; All authors have read and agreed to the published version of the manuscript.

Conflict-of-interest statement: The authors declare that they have no conflicts of interest.

Funding: This research received no external funding.

\section{References}

1 Díaz del Arco, C. et al. Are Borrmann's Types of Advanced Gastric Cancer Distinct Clinicopathological and Molecular Entities? A Western Study. Cancers 13, 3081 (2021).

2 Stojanovic, M. M., Rancic, N. K., Andjelkovic Apostolovic, M. R., Ignjatovic, A. M. \& Ilic, M. V. Trends of Stomach Cancer in Central Serbia. Medicina 57, 665 (2021).

3 Dassen, A. E. et al. Gastric cancer: decreasing incidence but stable survival in the Netherlands. Acta Oncologica 53, 138-142 (2014).

4 Siegel, R. L., Miller, K. D. \& Jemal, A. Cancer statistics, 2016. CA: a cancer journal for clinicians 66, 7-30 (2016).

5 Yaprak, G., Tataroglu, D., Dogan, B. \& Pekyurek, M. Prognostic factors for survival in patients with gastric cancer: Single-centre experience. Northern clinics of Istanbul 7, 146 (2020).

$6 \quad$ Ferlay, J. et al. Estimating the global cancer incidence and mortality in 2018: GLOBOCAN sources and methods. International journal of cancer 144, 1941-1953 (2019).

7 Safari, M., Mahjub, H., Esmaeili, H., Sadighi, S. \& Roshanaei, G. Estimating the treatment effect in patients with gastric cancer in the presence of Noncompliance. Gastroenterology and Hepatology from Bed to Bench (2021).

8 Maroufizadeh, S., Hajizadeh, E., Baghestani, A. R. \& Fatemi, S. R. Multivariate analysis of prognostic factors in gastric cancer patients using additive hazards regression models. Asian Pac J Cancer Prev 12, 1697-1702 (2011).

9 Farmanfarma, K. K., Mahdavifar, N., Hassanipour, S. \& Salehiniya, H. Epidemiologic Study of Gastric Cancer in Iran: A Systematic Review. Clinical and Experimental Gastroenterology 13, 511 (2020).

10 Talebi, A. et al. Survival analysis in gastric cancer: a multi-center study among Iranian patients. BMC surgery 20, 1-8 (2020).

11 Borumandnia, N. et al. Nomogram to Predict the Overall Survival of Colorectal Cancer Patients: A Multicenter National Study. International journal of environmental research and public health 18, 7734 (2021).

$12 \mathrm{Xu}, \mathrm{W}$. et al. Prediction model of tumor regression grade for advanced gastric cancer after preoperative chemotherapy. Frontiers in Oncology 11 (2021).

13 Faradmal, J., Talebi, A., Rezaianzadeh, A. \& Mahjub, H. Survival analysis of breast cancer patients using cox and frailty models. Journal of Research in Health Sciences $\mathbf{1 2}$, 127-130 (2012). 
14 Nilsaz-Dezfouli, H., Abu-Bakar, M. R., Arasan, J., Adam, M. B. \& Pourhoseingholi, M. A. Improving gastric cancer outcome prediction using single time-point artificial neural network models. Cancer informatics 16, 1176935116686062 (2017).

15 Niu, P.-H., Zhao, L.-L., Wu, H.-L., Zhao, D.-B. \& Chen, Y.-T. Artificial intelligence in gastric cancer: Application and future perspectives. World Journal of Gastroenterology 26, 5408 (2020).

16 Zhou, C. et al. A machine learning-based predictor for the identification of the recurrence of patients with gastric cancer after operation. Scientific reports 11, 1-7 (2021).

17 Zhu, S.-L., Dong, J., Zhang, C., Huang, Y.-B. \& Pan, W. Application of machine learning in the diagnosis of gastric cancer based on noninvasive characteristics. Plos one 15, e0244869 (2020).

18 Almazar, A. E., Penfield, J. D., Saito, Y. A. \& Talley, N. J. Survival times of patients with Menetrier's disease and risk of gastric cancer. Clinical Gastroenterology and Hepatology 19, 707-712 (2021).

19 Liang, X. et al. Development of a Clinical Nomogram for Prediction of Response to Neoadjuvant Chemotherapy in Patients with Advanced Gastric Cancer. (2021).

20 Zhou, X. et al. A novel nomogram for predicting survival of patients with poorly differentiated gastric adenocarcinoma. TRANSLATIONAL CANCER RESEARCH 10, 886-898 (2021).

21 Jalali, A., Alvarez-Iglesias, A., Roshan, D. \& Newell, J. Visualising statistical models using dynamic nomograms. PloS one 14, e0225253 (2019).

22 Kawai, K. et al. Nomogram predicting survival after recurrence in patients with stage I to III colon cancer: a nationwide multicenter study. Diseases of the Colon \& Rectum 61, 1053-1062 (2018).

23 Song, K. Y., Park, Y. G., Jeon, H. M. \& Park, C. H. A nomogram for predicting individual survival of patients with gastric cancer who underwent radical surgery with extended lymph node dissection. Gastric Cancer 17, 287-293 (2014).

24 Liu, Y. \& De, A. Multiple imputation by fully conditional specification for dealing with missing data in a large epidemiologic study. International journal of statistics in medical research 4, 287 (2015).

25 Gao, Z. et al. A nomogram for prediction of stage III/IV gastric cancer outcome after surgery: a multicenter population-based study. Cancer medicine 9, 5490-5499 (2020).

26 Mu, G.-C. et al. Application value of nomogram and prognostic factors of gastric cancer patients who underwent D2 radical lymphadenectomy. BMC gastroenterology 19, 1-8 (2019).

27 Wang, J. et al. Nomogram-based prediction of survival in unresectable or metastatic gastric cancer patients with good performance status who received first-line chemotherapy. Annals of translational medicine 8 (2020).

28 Han, D.-S. et al. Nomogram predicting long-term survival after d2 gastrectomy for gastric cancer. Journal of clinical oncology 30, 3834-3840 (2012).

29 Lu, J. et al. Development and external validation of a nomogram to predict recurrencefree survival after R0 resection for stage II/III gastric cancer: an international multicenter study. Frontiers in oncology 10 (2020).

30 Yin, Q. H. et al. A Nomogram Based on Preoperative Clinical Bio-Indicators to Predict 5-year Survivals for Patients with Gastric Cancer After Radical Gastrectomy. Cancer management and research 12, 3995 (2020). 
31 Chen, J. et al. A clinical model to predict distant metastasis in patients with superficial gastric cancer with negative lymph node metastasis and a survival analysis for patients with metastasis. Cancer medicine 10, 944-955 (2021).

32 Fang, W.-L. et al. The risk factors of lymph node metastasis in early gastric cancer. Pathology \& Oncology Research 21, 941-946 (2015).

33 Yang, H.-J. et al. Predictors of lymph node metastasis in patients with non-curative endoscopic resection of early gastric cancer. Surgical endoscopy 29, 1145-1155 (2015). 